\title{
Design of Deformation Monitoring Terminal System Based on STM32
}

\author{
Kaihua $\mathrm{Xu}^{1}$, Di Zhang ${ }^{2}$, Yuhua $\mathrm{Liu}^{2}$, Ke Xu², Yuanhao Xi ${ }^{3}$ \\ 1.College of Physical Science and Technology, Central China Normal University, Wuhan, China \\ 2.School of Computer, Central China Normal University, Wuhan, China \\ 3.Institute of Information, Liaoning University, Shenyang, China \\ Email: xukh@phy.ccnu.edu.cn, zhd87@hotmail.com,yhliu@mail.ccnu.edu.cn \\ Correspondence author: Yuhua Liu
}

\begin{abstract}
One monitoring Terminal system based on STM32 is introduced in this paper. The system used STM32 as core controller, GPIO and USART as control and communication channel. GPS, GPRS, wired transmission and power section together makes up the system motherboard. The design has been tested and been applied in geology disaster monitoring. Results show that it is adapted to the harsh environment outdoors and long term working conditions.
\end{abstract}

Keywords—deformation monitoring; terminal system; hardware design; receive and transmit system design; data transmission

\section{INTRODUCTION}

Natural disaster, especially the geological disaster such as debris flow and earthquake, cause enormous loss both economics and labor [1]. According to Brookings, 2010, there are 390 thousands of people died while 294 million people that suffered the natural calamities. As the Ministry of China Land and Resources said, there were 15644 geological disasters, which took away 245 person's lives and 4 billion Yuan in 2011. There were about 334 thousands of nature disasters, which took 14270 persons' lives away and direct losses summed up to 62, 2540 billion Yuan from 1998 to now. In this case, it is significant and urgent to avoid this disaster by monitoring or other methods.

Nature disaster monitoring technology utilizes complex technological methods. Deformation monitoring is one of the most important parts as it can identify the landslide. In traditional methods of nature disaster monitoring methods, reshuffled signal collection is accomplished by carrying monitoring instruments [2], which brings great work load with long operation cycle while worse real-time characteristic. GPS technology cooperated with wireless or wired transmission technology is noticed in deformation monitoring nowadays. GPS with wireless sensor networks [2], GPS with wired internet [3] has been proposed by some researchers and companies for monitoring. However, if we can combine both wired and wireless internet for transmission, the deformation monitoring system would be suitable for durable time monitoring with intelligence.

In this paper, one method of deformation monitoring terminal system based on STM32 processor is proposed. GPS module, GPRS module, wired transmission section and core controller based on STM32 makes up the motherboard. Scene generator with high storage battery provides the UPS. Both of the motherboard and battery are protected in stainless steel box to resist the severely outdoor condition. It can monitor the geology deformation by planting series of them in destination area. GPS data transmitted through wireless network to remote center is used for pseudo realtime calculating if there is good condition of mobile signals. Otherwise, it will transmit through wired network. The remote center can get accuracy 3-Dimensional coordinates information of the area with little delay by calculating them [4]. As the backup of transmission method, battery and scene generator, once planted in the destination area, the system will be effective for much durable time.

Detail hardware design is discussed in section II. General design and scope of the system is the first. The scenery integration power system can provide long term uninterrupted power. Afterwards, Specific parts of GPS and GPRS and their circuit designs are reviewed. Section III is the software design. The flow chart of general design is introduced in the beginning. Afterwards, flow control of write and read of GPS data and buffer is discussed. CRC32 and ring buffer are used to provide reliable and steady data structure and transmission. Test and result of the system is in section IV, which includes the test environment, devices set and result. Results analysis also included in this section. Section $\mathrm{V}$ is the summery about the terminal design and results of the test.

\section{HARDWARE DESIGN OF DEFORMATION MONITORING TERMINAL SYSTEM}

Terminal system is the outdoor machine of the Disaster Deformation Monitoring System, which consisted by the outdoor system and remote center. It will get the accuracy 3Dimensional coordinates information of the monitoring area by calculating GPS data that transmitted to the remote center. Detail deformation condition can gain in this method. Meanwhile, battery voltage and working condition data of terminal system will transmit to the remote center to ensure the working condition of the machine. Bad condition will reflect on the screen of remote center if antenna goes wrong or voltage lower than the working demand.

Terminal system can decide to use GPRS or Wired internet for transmission according to the network environment of the specific area. GPRS is the first choice when there is good cover of CMCC signal because of the security guarantee. Wired network will be used in case of the bad condition of GPRS.

Motherboard, GPS and GPRS antenna, Scene generator and packing together consist of the Terminal system. CPU 
based on STM32, communication module, GPS and GPRS module with specific antenna of each consist of the host. Detail block diagram of hardware design and interfaces is shown in Figure 1.

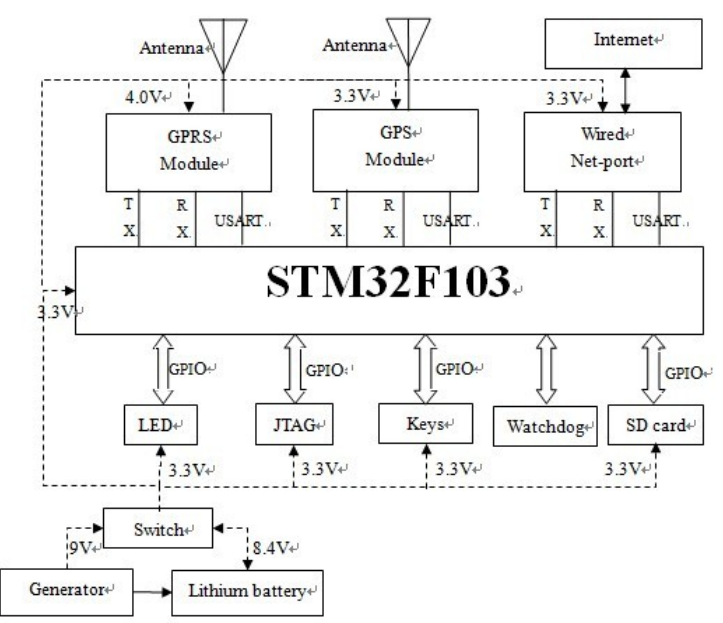

Figure 1 Block diagram of hardware design and interfaces of deformation monitoring terminal system.

MCU is the STM32F103 series with voltage of 3.3V. The constant working voltage is supplied by AMS1117, which makes the voltage accuracy within $1.5 \%$ [5]. The MCU has dominant frequency of $72 \mathrm{MHz}$ with calculating ability as much as 90DMIPS. It is one of the series of microprocessor of ARM-Cortex M3, based on ARMv7 architecture. It can satisfy the complex requirement of embedded systems with MMU to realize the virtual memory scheme. And because of the stable work condition and fast repercussion, it can be used in the environment which needs strict real-time.

The ARM-Cortex M3 core utilizes hybrid Thrumb-2 16/32 instruction set, which switching of Thrumb and ARM set is not needed while working. In this case, processing efficiency is greatly increased. The bit-band operate can process every signal bit. The read/write of $\mathrm{I} / \mathrm{O}$ register is optimized with much faster speed of bit operating in this method. While in developing period, the debug can be achieved by SWD, which only need two pins to connect with the MCU. It also supports 3-stage pipeline of fetch, decode, execute, hardware division and single cyclic multiplication with very low consumption [6].

GPS module and wired net-port are connected to the MCU by USART with working voltage of 3.3V. GPRS module works at the voltage of $4.0 \mathrm{~V}$, which corresponds by USART, too. While EN pin is low power, MC29301 will have no output to the module. The GPS module can be powered only in the case that EN pin is in the condition of high power. GPS board provides hardware reset through generate IO pins to restart in the event of data congestion or any other proruption conditions. It is also provided with steady DC voltage by PMIC. GPS antenna with low noise amplify module built in adopts feedback contact, which filtering noise via multi stage filter, joints in GPS board through TNC pins. SIM cassette has 6 pins, two of them used for power supplying, which current is filtered by $220 \mathrm{nF}$ capacitance in case of any interface pulse. IO pin communicates with GPRS module through a $10 \mathrm{k} \Omega$ pull up resistor. GPIO can control the net-port for configuration, reset, controlling function IO [7], which works in full or half duplex model and can be configured through webpage builtin the web server.

Storage area is selected as the boot sector with BOOT. It will send the voltage of MCU information back to remote center through USART together with GPS data. Watchdog is utilized in case of fleeting of the program. There are some other design methods to ensure the robust. Such as LED lights that communicate with MCU through GPIO to display the machine condition of power on, receiving GPS data, uploading GPS data, power off condition of the system. JTAG is the interface used for program download. Control commands of the machine can achieve by pressing the buttons on the system machine. SD card is used for storage of GPS signal for post processing in case. All of them communicate with MCU by GPIO with working voltage of 3.3V.

High capacity lithium battery and Scenery generator provide the system power. Switch circuit is utilized to decide whether the battery or scenery generator should be used by estimating the steady and voltage of them to ensure ongoing power. While there is only one of them used, switch circuit will select that one. However, if all of them with stable voltage join in, the scenery will charge the battery [8]. Circuit connection is shown as in Figure 2.

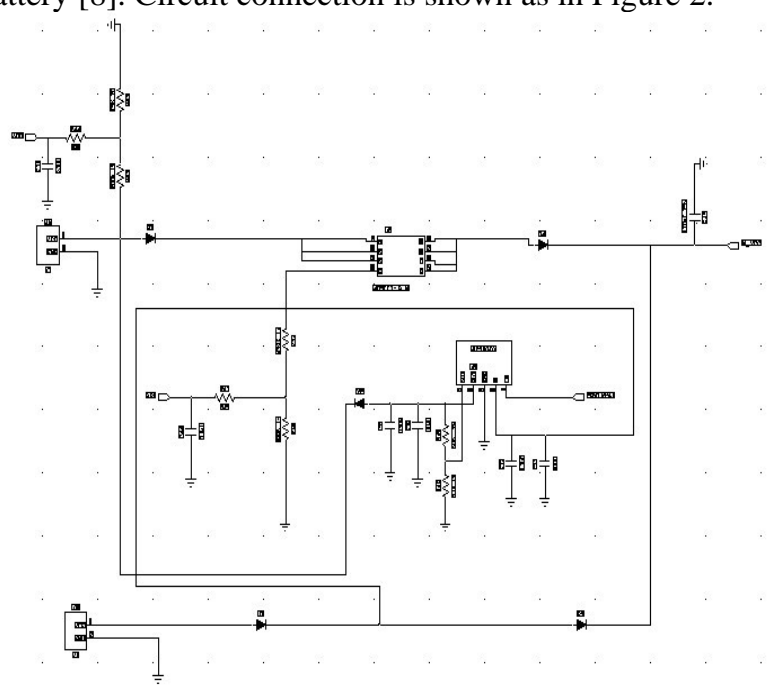

Figure 2 Circuit connection of the power and controller

P channel power MOSFET is the power switch here. A number of Schottky diodes are used for rectifying. If U19 with voltage of $9 \mathrm{~V}$ and $\mathrm{U} 3$ with voltage of $8.4 \mathrm{~V}$ connect into the circuit together, it will make the MOS diode cut off. The battery will be charged constantly through 2930X, step down regulator, when voltage of U3 is lower than $8.4 \mathrm{~V}$. MOS diode will conduct and voltage of battery will export through two diodes in case of only battery joined. Afterwards, enable pin of $2930 \mathrm{X}$ is connected with MCU to 
monitoring the voltage of battery to decide whether it is charging or not. Capacitance of C45 is used to smooth the voltage in case of pluse disturbing.

\section{Software Design of Deformation Monitoring TERMINAL SYSTEM}

The software development is based on STM32F103X series Firmware and AT commands of SIMCOM is used. The command of configuration of clock, independent watchdog, interrupt are mainly utilized. USART1, 2, 3 pins are configured for communication of GPS, GPRS, voltage information of MCU and wired net-port. LED signal lights are selected for working conditions indication with its flash and other states. For instance, flash one time stands for communication transmission prepared, 5 times flash means GPS satellites online, two lights without any flash on stand for terminal system working normally. Watchdog is utilized to monitor the program to ensure the continuous working in the unattended open air [9]. Flow chart of the program is shown in Figure 3.

The baud rate of GPS module should be $19200 \mathrm{HZ}$ to match transmission rate because default rate is $9600 \mathrm{HZ}$. Ephemeris and epoch data, whose output time decided by configuration files, should provide to remote center for calculating. Ring buffer, one kinds of buffer with good data utilization rate that widely used in embedded system devices is used as our data buffer. Its input and output location can be figured out by base address and offset. Data processing mission is used for receiving, check and write from the buffer. Those data that cannot pass through CRC32 verify and blank date will be abandoned. While receiving, start bit is used to decide whether the frame is coming or not. GPS date verification is accomplished by identify the Message ID and Message Type. Because of the header length of ephemeris and epoch both are 28 bytes, although the header length can be changed according to the GPS module introduction, the header length is configured to 28 bytes. The flow chart of receiving is shown in Figure 4.

While transmitting, GPRS is selected as the first solution. If the GPRS environment is not so good for transmission, the system can select wired internet. Before transmission, baud rate should be configured to match the transmit rate. After that, the system will read the buffer for data and package them into TCP/IP protocol stack. GPS data transmission can be realized by configured the AT commands. Detail transmission flow is shown as follows in Figure 5.

Starting bit, message ID and type are used to decide whether the data is coming to estimate whether it is GPS data or not, and data volume and check bit are utilized to complete one frame receiving. Trigger will strike if GPS data is coming. The useful data will be written into the GPS data buffer while the useless frame will be abandoned.

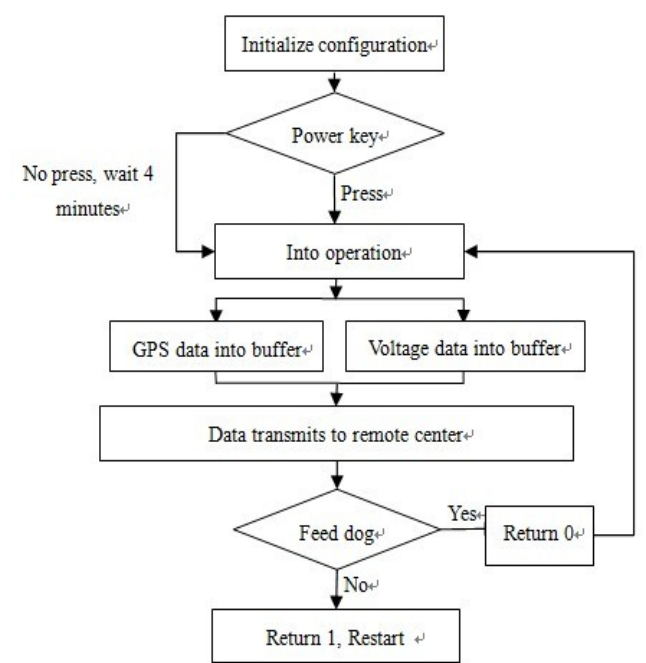

Figure 3 Flow chart of general program about terminal system

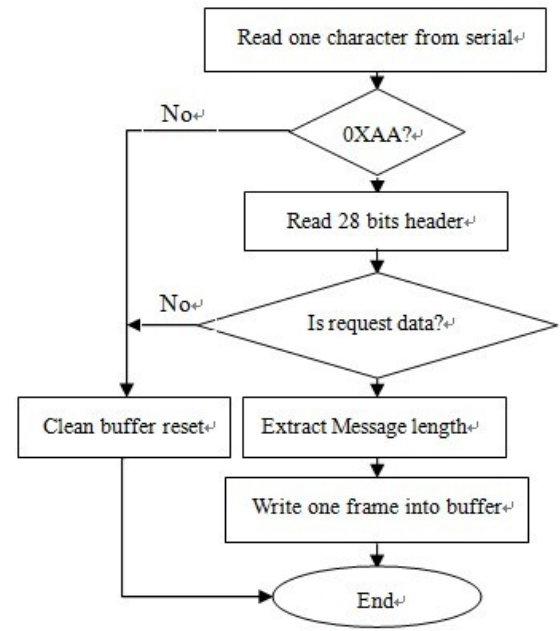

Figure 4 Flow of receiving about GPS data

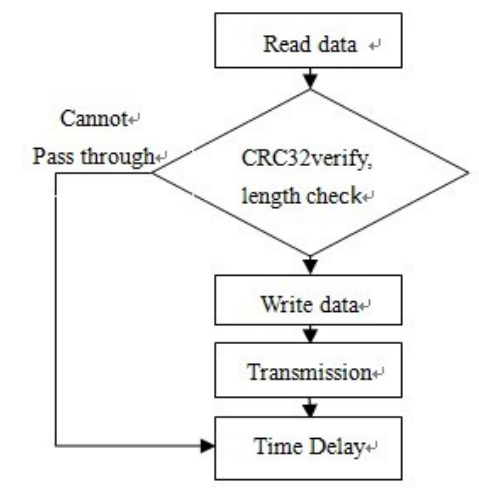

Figure 5 Detail transmission flow of the system

\section{APPLICATION TEST OF TERMINAL SYSTEM}

According to standard of GPS surveying GB/T 183142009 and CH/T 2008-2005, GPS receiver should assign in environment without block of obstacle and mental 
conductor $15^{\circ}$ above. In this test, there are 3 terminal systems, one used as base station and two other as the monitoring mobile station. GPS antennas are placed in forced centering point in the test. Detail test scheme is shown in Table 1.

As the result relays on GPS receive capacity and processing ability of terminal system and reliability of GPRS transmission, and because of the monitoring accuracy of deformation monitoring, for the remote center, the integrity data should be more than $90 \%$ transmitted by the terminal system. Test illustrates that terminal system devise is reliable. Specific test result is shown in table 2 .

Because of the precision might be affected by base line used while monitoring, to verify the accuracy of different base line, the long and short base line test are required. In this test, there are also three machines, one used as base station, two others used as monitoring terminal system. Six forced centering points, named as J1, J2, J3, J4, J5, J6 with $\mathrm{J} 2$, J3, J4 used as short base line test points, J1, J5, J6 used as long base line test points are used in the test. 2.5 hours' data are calculated to gain the length of each base line with frequency of receiving of GPS data set as $1 \mathrm{HZ}$ [10]. The test result is shown as in table 3 .

\begin{tabular}{|c|c|c|}
\multicolumn{3}{|c|}{ Table 1 Detail test scheme } \\
\hline Devices & Test duration(h) & GPS frequency(HZ) \\
\hline Base station & 3 & 1 \\
\hline Mobile station 1 & 3 & 1 \\
\hline Mobile station 2 & 3 & 1 \\
\hline
\end{tabular}

Table 2 Test result of terminal system

\begin{tabular}{|c|c|c|c|}
\hline Devices & $\begin{array}{c}\text { Theory epoch } \\
\text { number }\end{array}$ & $\begin{array}{c}\text { Actual epoch } \\
\text { number }\end{array}$ & Data integrity \\
\hline Base station & 10800 & 10751 & $99.54 \%$ \\
\hline Mobile station 1 & 10800 & 10766 & $99.68 \%$ \\
\hline Mobile station 2 & 10800 & 10743 & $99.47 \%$ \\
\hline
\end{tabular}

Table 3 Base line test result

\begin{tabular}{|c|c|c|c|c|c|}
\multicolumn{7}{c|}{ Table 3 Base line test result } \\
\hline Base & Monitor & $\begin{array}{c}\mathrm{X} \\
\text { Axis(m) }\end{array}$ & $\begin{array}{c}\text { Y } \\
\text { Axis(m) }\end{array}$ & $\begin{array}{c}\mathrm{Z} \\
\text { Axis(m) }\end{array}$ & $\begin{array}{c}\text { Base } \\
\text { Line }\end{array}$ \\
\hline J5 & J1 & -432.828 & -172.681 & -33.234 & 467.187 \\
\hline J5 & J6 & -1984.025 & 2088.322 & -4639.333 & 5460.847 \\
\hline J1 & J6 & -1551.188 & 2260.984 & -4606.104 & 5360.450 \\
\hline J3 & J2 & -133.816 & -51.456 & -13.483 & 144.001 \\
\hline J3 & J4 & 178.416 & 68.609 & 17.967 & 191.995 \\
\hline J3 & J5 & 142.891 & 61.197 & 4.011 & 155.496 \\
\hline J2 & J4 & 312.232 & 120.064 & 31.449 & 335.996 \\
\hline J2 & J5 & 276.708 & 112.651 & 17.493 & 299.272 \\
\hline J4 & J5 & -35.523 & -7.413 & -13.956 & 38.880 \\
\hline
\end{tabular}

Table 4 Measuring error of the test

\begin{tabular}{|c|c|c|c|}
\multicolumn{4}{|c|}{ Table 4 Measuring error of the test } \\
\begin{tabular}{|c|c|c|c|}
\hline \multicolumn{2}{|c|}{ Short Base Line } & \multicolumn{2}{c|}{ Long Base Line } \\
\hline Base Station & $\begin{array}{c}\text { Measuring } \\
\text { Error(mm) }\end{array}$ & $\begin{array}{c}\text { Base } \\
\text { Station }\end{array}$ & Measuring Error(mm) \\
\hline J2 & 0.7 & J1 & 1.6 \\
\hline J3 & 2.5 & J5 & 1.6 \\
\hline J4 & 2.5 & J6 & 7 \\
\hline
\end{tabular}
\end{tabular}

The measuring error can be gain by compare the standard base line length of different base point, which is shown as table4. In the test, J6 located in an environment with trees and buildings, which has negative effect to the accuracy. Precision of the other points with accuracy in mm level, shows that the designed system can be used for accuracy deformation monitoring.

\section{CONCLUSION}

The STM32 processor together with GPS and GPRS modules and their antennas are used in the deformation monitoring terminal system, which can be used in nature disaster deformation monitoring especially for mud rock flow and dam deformation monitoring. It can provide for long time and practically real time monitoring for its durable power system and robust design of hardware and software. The results of test prove that it is reliable and practical for the monitoring demand.

\section{ACKNOWLEDGEMENT}

This work was supported in part by the Ministry of Science and Technology of P.R.C and in part by the Ministry of Finance of P.R.C, under grant 2012-DHFWY10056.

\section{REFERENCES}

[1] Papageoriou E, Foumelis M and Parcharidis I, Long and Short Term Deformation Monitoring of Santorini Volcano: Unrest Evdience by DinSAR Analysis, IEEE J. Selected Topics in Applied Earch Observations and Remote Sensing, Vol.5. New York, 2012, 15311537

[2] Zhu bin and Xia Kai, Design of Subsidence Monitoring System based on Wireless Sensor Networks, 6thWiCOM, IEEE, Chengdu, China, Sept. 2010, 1-4

[3] Meng, X, Dodson, A. H, Roberts, G. W and Andreotti, M, Prototype Internet RTK GPS for Bridge Deformation Monitoring, J. Suvery Review. Vol.38, 2006, 348-357

[4] Jun-zheng Li, Jin-ming Hao, Jian-wen Li, Jun-yi Li and Zhi-qiao Chang, The Principle of GPS Virtual Reference Station and the Testing and Analysis of Actual Measured Accuracy. J.Engineering of Surveying and Mapping. Vol.18. Harbin, China, 2009, 21-27

[5] Yong-hong Wang, Wei Xu and Li-ping Hao. Theory and Practise of STM32 Series of CORTEX-M3 Micro Processor. Beihang Press, Beijing, 2008

[6] Lei Xu, Guan Limin and Bai Yunting, Design on Remote Intelligent Terminal Unit of Industrial Enternet Based on STM32, J. Advanced Materials Research, Vlo.466. Switzerland, 2012, 1167-1171

[7] Kaihua Xu, Jun Zhou, Yuhua Liu and shuyun Dong, Signal Integrity Research of High Speed Circuit of Embedded System, FCST, IEEE Computer Society, Shanghai, China, 2009, 473-477.

[8] Kaihua $\mathrm{Xu}$, Jian Chen, Yuhua Liu and Rui Wang, Implementation of handheld remote sense image calibration system software, ICFCC, IEEE Computer Society, Wuhan, China, 2010, 664-668

[9] Ming-gui Liu, Jian Yu and Hao Liang, Wireless Geotechnical Engineering Acquisition System Based on STM32. J. Instrument Technique and Sensor. Vol.5.Shenyang, China, 2010, 95-97

[10] Hu Congwei and Liu Dajie, GPS Surveying Based on GPS Reference Station Network, J. Modern Surveying and Mapping, Vol.26.Nanjing, China, 2003, 10-13 